Supporting Information For

\title{
The Doping Effect of 13-Atoms Iron Clusters on Water Adsorption and O-H Bond Dissociation
}

Hongchao Zhang, Chaonan Cui*, and Zhixun Luo*

State Key Laboratory for Structural Chemistry of Unstable and Stable Species, Institute of

Chemistry, Chinese Academy of Sciences, Beijing 100190; and University of Chinese Academy of Sciences, Beijing 100049, China.

*Correspondence. Email: chncui@iccas.ac.cn (CC); zxluo@iccas.ac.cn (ZL) 


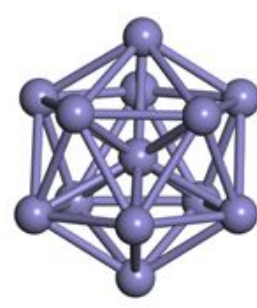

0

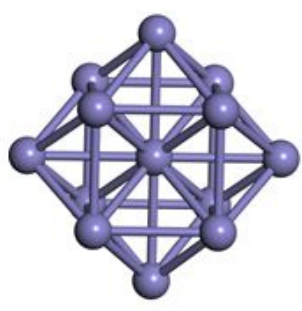

$1.16 \mathrm{eV}$

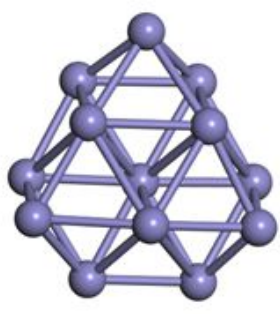

$1.65 \mathrm{eV}$

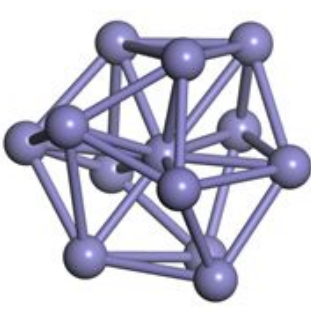

$1.64 \mathrm{eV}$

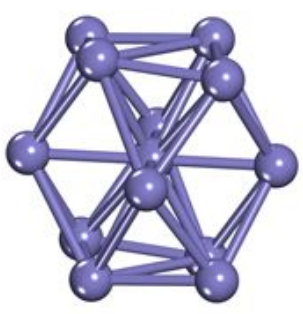

$1.12 \mathrm{eV}$

Figure S1. The optimized configurations and relative energies of $\mathrm{Fe}_{13}$ isomers.

a)
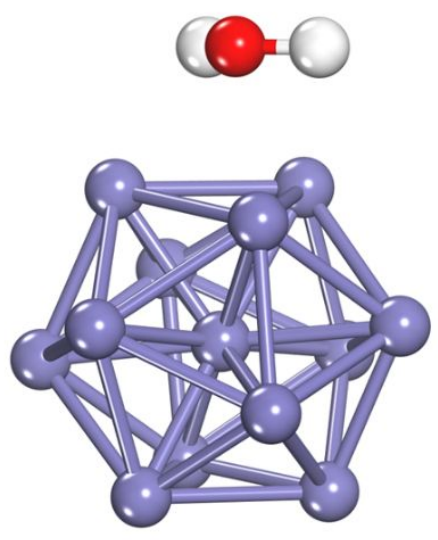

b)
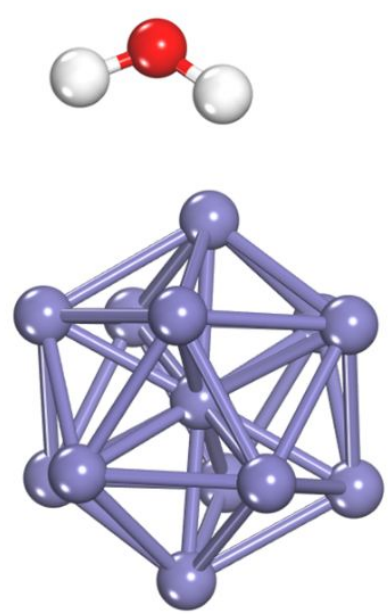

c)
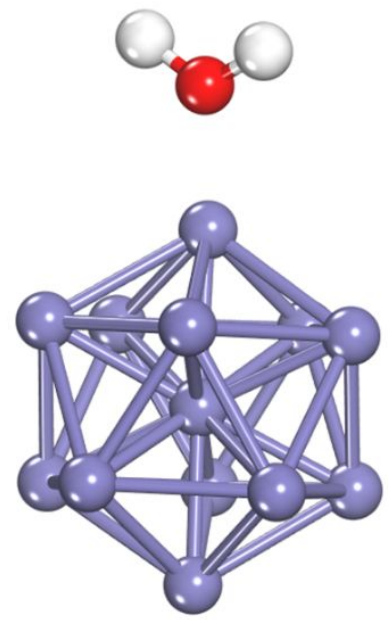

Figure S2. The initial orientations for water molecule relative to the clusters, with the $\mathrm{O}-\mathrm{H}$ bonds a) parallel, b) upward and c) downward to the shell surface. 


\section{a)}

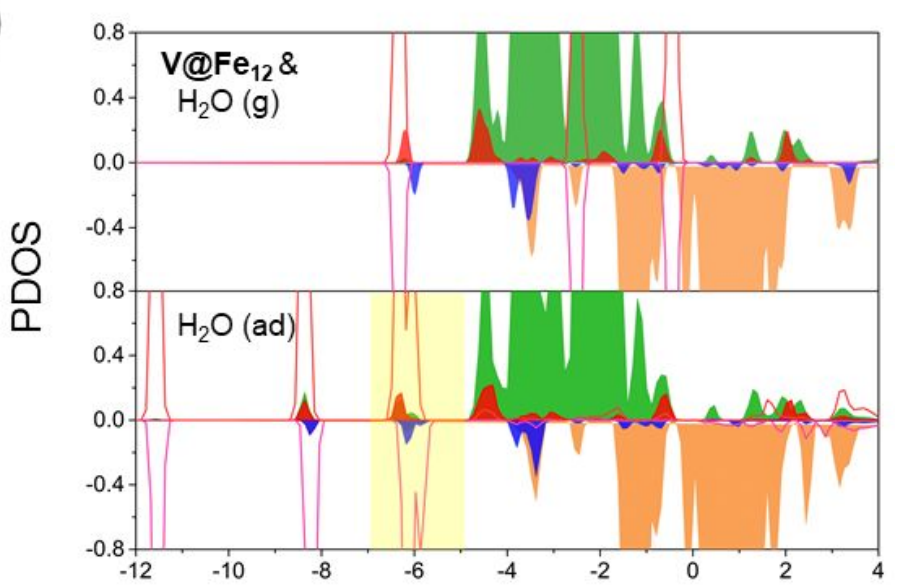

$E-E_{F}(e V)$

b)

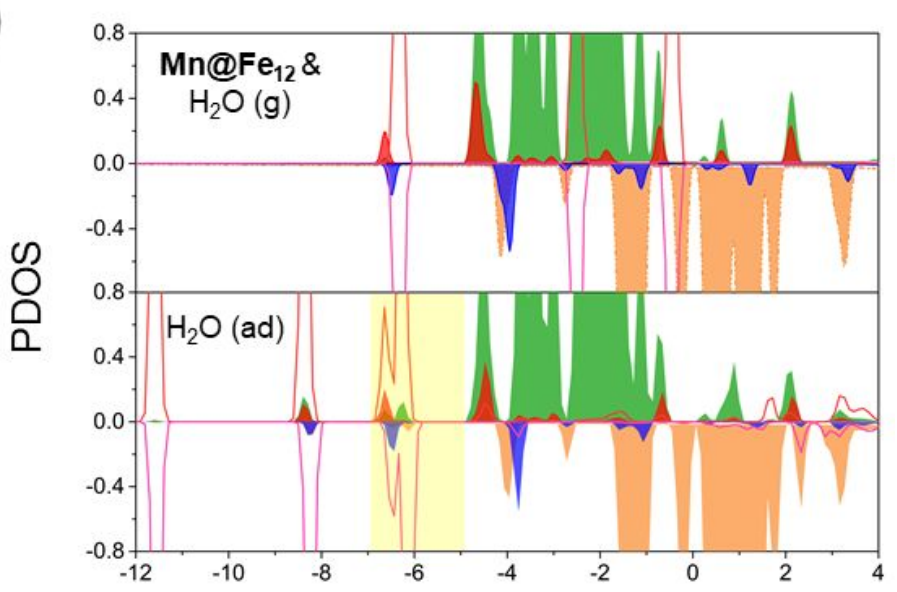

$E-E_{F}(e V)$

c)

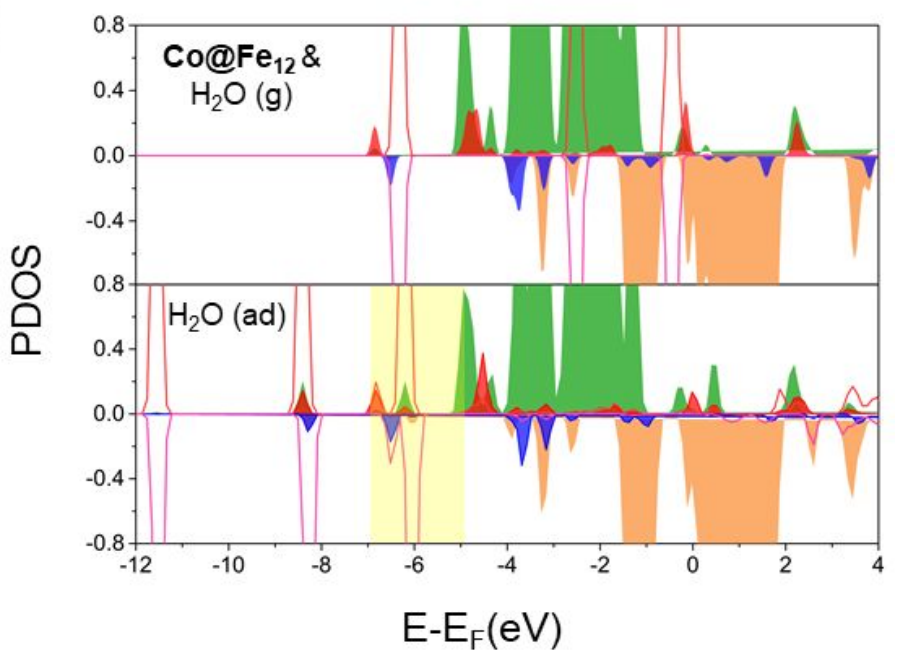

Figure S3. The PDOS for the $\mathrm{H}_{2} \mathrm{O}$ and the adsorbed Fe atom. The area filled with green $(\alpha)$, orange $(\beta)$, $\operatorname{red}(\alpha)$ and blue $(\beta)$ represent the $d$ and s orbital of Fe atom, respectively. Red and purple solid line represent the $\mathrm{p}$ orbital of $\mathrm{O}$ atom. The Fermi level is set to be zero. 


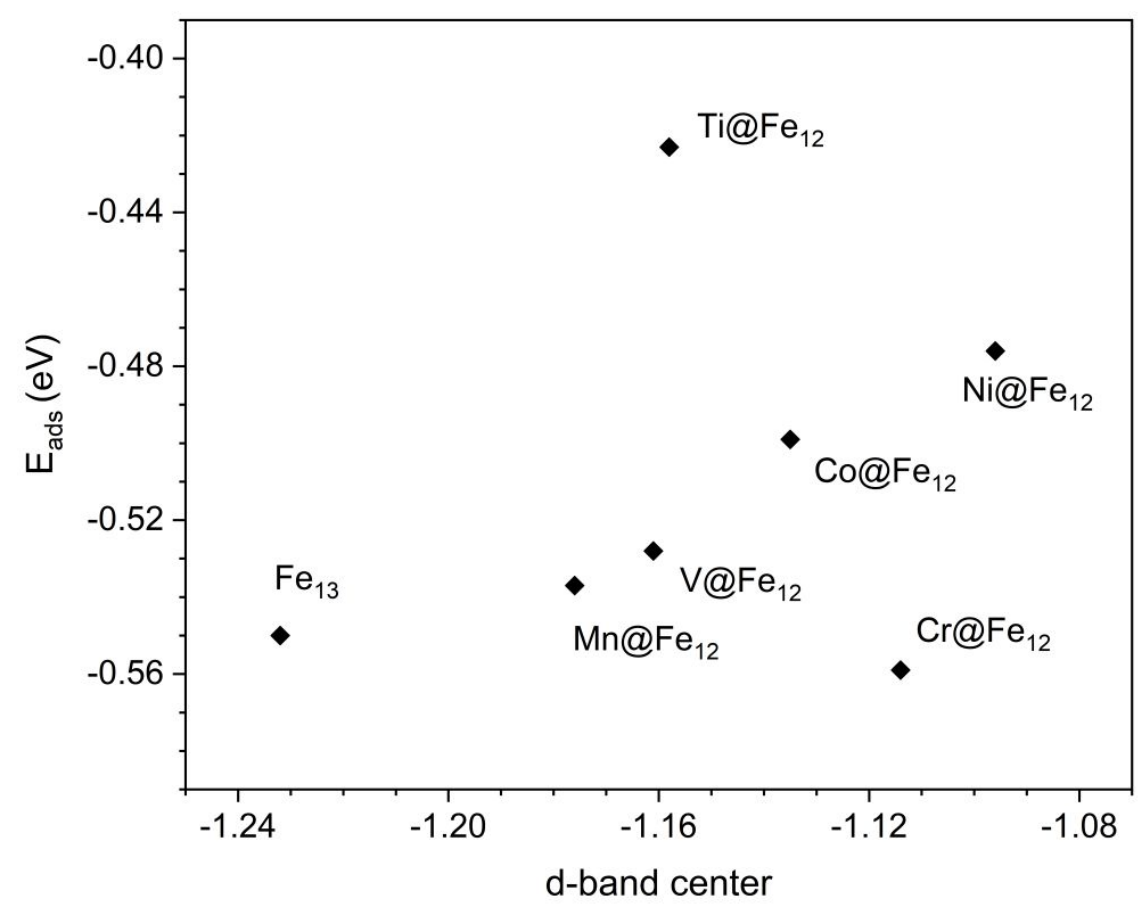

Figure S4. The water adsorption energy vs the d-band center of the $\mathrm{X} @ \mathrm{Fe}_{12}$ clusters. The Fermi level is set to be zero.

a)

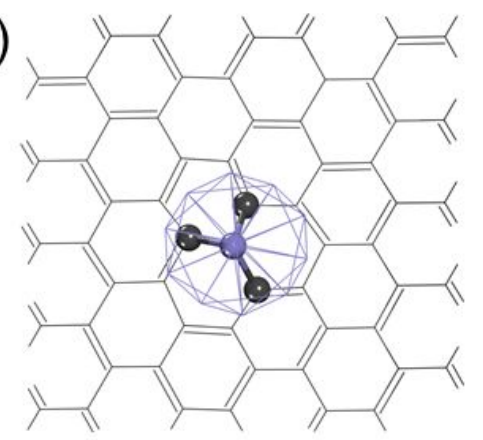

$\mathrm{H} 1$ b)

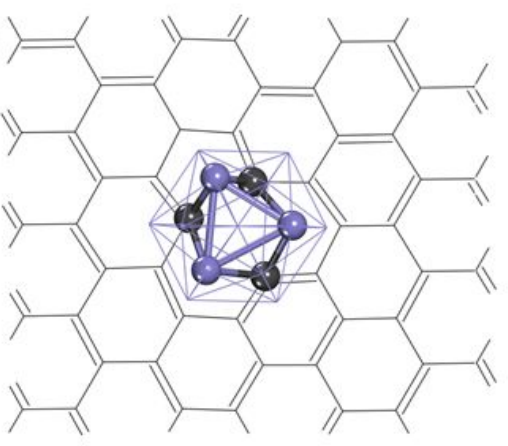

H3 c)

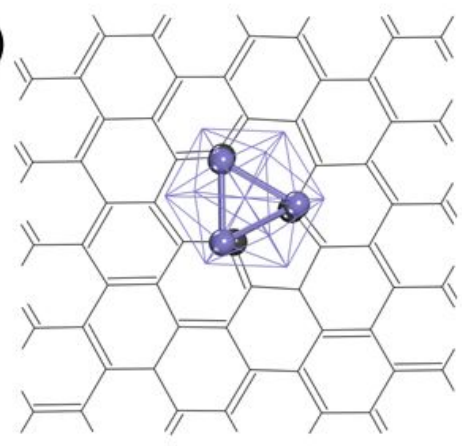

T3

\begin{tabular}{cccc}
\hline & H1 & H3 & T3 \\
\hline $\mathrm{Ti} @ \mathrm{Fe}_{12}$ & -5.92 & -3.52 & -3.00 \\
$\mathrm{Fe}_{13}$ & -6.72 & -4.07 & -3.47 \\
$\mathrm{Ni} @ \mathrm{Fe}_{12}$ & -6.67 & -4.25 & - \\
\hline
\end{tabular}

Figure S5. Three different initial landing modes of $\mathrm{X} @ \mathrm{Fe}_{12}$ and corresponding energies on defective graphene with a monovacancy. As for $\mathrm{T} 3$ of $\mathrm{Ni@} \mathrm{Fe}_{12}$, it turns to $\mathrm{H} 3$ after optimization. 


\begin{tabular}{cccc} 
& \\
\hline
\end{tabular}

Figure S6. The Bader charge analysis of $\mathrm{X} @ \mathrm{Fe}_{12} / \mathrm{DG}(\mathrm{X}=\mathrm{Ti}, \mathrm{Fe}, \mathrm{Ni})$. 
a)

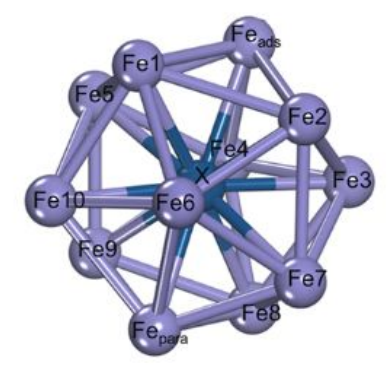

b)

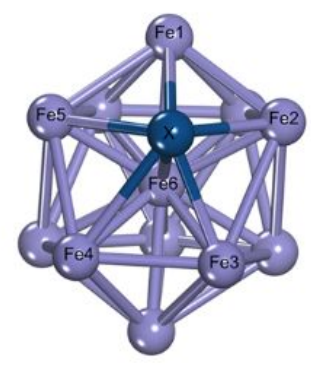

c)

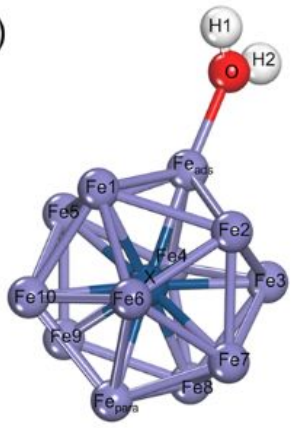

Figure S7. The corresponding number of $\mathrm{Fe}$ and $\mathrm{H}$ atoms for a) bare $\mathrm{X} @ \mathrm{Fe}_{12}, \mathrm{~b}$ ) bare $\mathrm{X}-\mathrm{Fe}_{12}$ and c) $\mathrm{X} @ \mathrm{Fe}_{12}-\mathrm{H}_{2} \mathrm{O}$ clusters of Table S1, Table S2 and Table S4.

a)

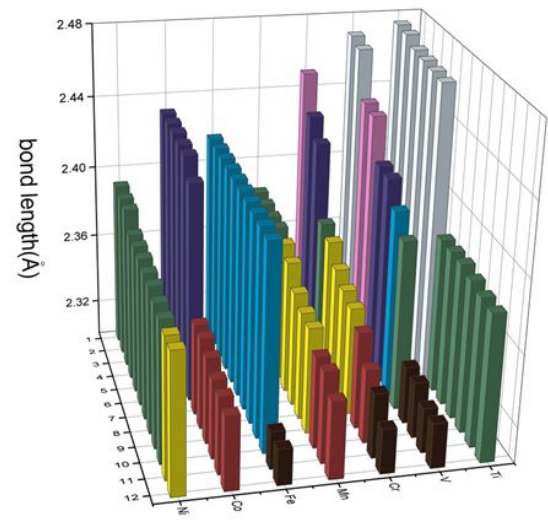

b)

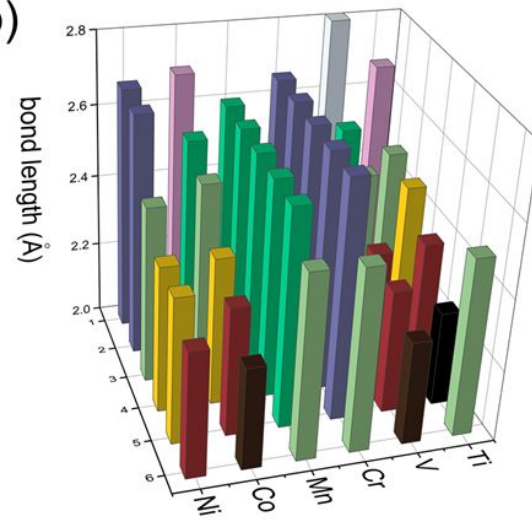

Figure S8. The Fe-X bond lengths of $\mathrm{X} @ \mathrm{Fe}_{12}$ (a) and X-Fe ${ }_{12}$ (b) clusters, details in Table $\mathrm{S} 1$ and S2.

Table S1. Bond length (in $\AA$ ) between the doping atom X and Fe atoms in X@Fe $\mathrm{Fe}_{12}$ systems. The corresponding number of $\mathrm{Fe}$ atom and $\mathrm{H}$ atom can be seen in Figure $\mathrm{S} 7$ (a).

\begin{tabular}{cccccccc}
\hline $\begin{array}{c}\text { Doping } \\
\text { atom }\end{array}$ & $\mathrm{Ti}$ & $\mathrm{V}$ & $\mathrm{Cr}$ & $\mathrm{Mn}$ & $\mathrm{Fe}$ & $\mathrm{Co}$ & $\mathrm{Ni}$ \\
\hline $\mathrm{Fe} 1$ & 2.47 & 2.40 & 2.35 & 2.36 & 2.42 & 2.35 & 2.38 \\
$\mathrm{Fe} 2$ & 2.47 & 2.33 & 2.33 & 2.38 & 2.32 & 2.35 & 2.39 \\
$\mathrm{Fe} 3$ & 2.39 & 2.33 & 2.36 & 2.38 & 2.42 & 2.43 & 2.38 \\
$\mathrm{Fe} 4$ & 2.47 & 2.44 & 2.37 & 2.38 & 2.42 & 2.43 & 2.38 \\
$\mathrm{Fe} 5$ & 2.47 & 2.47 & 2.43 & 2.35 & 2.42 & 2.43 & 2.39 \\
$\mathrm{Fe} 6$ & 2.38 & 2.47 & 2.38 & 2.38 & 2.42 & 2.43 & 2.38 \\
$\mathrm{Fe} 7$ & 2.47 & 2.44 & 2.45 & 2.36 & 2.42 & 2.43 & 2.38 \\
$\mathrm{Fe} 8$ & 2.47 & 2.33 & 2.38 & 2.36 & 2.42 & 2.35 & 2.38 \\
$\mathrm{Fe} 9$ & 2.48 & 2.33 & 2.36 & 2.38 & 2.32 & 2.34 & 2.38 \\
$\mathrm{Fe} 10$ & 2.39 & 2.41 & 2.37 & 2.37 & 2.42 & 2.43 & 2.38 \\
$\mathrm{Fe}$ & 2.39 & 2.42 & 2.42 & 2.34 & 2.42 & 2.35 & 2.39 \\
$\mathrm{Fe}{ }_{\text {ads }}$ & 2.39 & 2.42 & 2.34 & 2.36 & 2.42 & 2.35 & 2.38 \\
\hline
\end{tabular}


Table S2. Bond length between doping atom X and Fe atom in X-Fe12 systems. The corresponding number of $\mathrm{Fe}$ atom and $\mathrm{H}$ atom can be seen in Figure $\mathrm{S} 7 \mathrm{(b)}$.

\begin{tabular}{ccccccc}
\hline Doping atom & $\mathrm{Ti}$ & $\mathrm{V}$ & $\mathrm{Cr}$ & $\mathrm{Mn}$ & $\mathrm{Co}$ & $\mathrm{Ni}$ \\
\hline $\mathrm{Fe} 1$ & 2.26 & 2.35 & 2.67 & 2.61 & 2.36 & 2.41 \\
$\mathrm{Fe} 2$ & 2.38 & 2.37 & 2.67 & 2.61 & 2.41 & 2.41 \\
$\mathrm{Fe} 3$ & 2.47 & 2.52 & 2.67 & 2.61 & 2.54 & 2.49 \\
$\mathrm{Fe} 4$ & 2.50 & 2.58 & 2.67 & 2.61 & 2.59 & 2.67 \\
$\mathrm{Fe} 5$ & 2.68 & 2.82 & 2.67 & 2.61 & 2.70 & 2.68 \\
$\mathrm{Fe} 6$ & 2.50 & 2.29 & 2.51 & 2.52 & 2.28 & 2.35 \\
\hline
\end{tabular}

Table S3. Water adsorption onto X@ $\mathrm{Fe}_{12}, G a{ }^{*}$ indicates gas phase water.

\begin{tabular}{|c|c|c|c|c|c|c|c|}
\hline \multirow{2}{*}{ Doping-atom } & \multicolumn{3}{|c|}{ Bond length $(\AA)$} & \multirow{2}{*}{$\begin{array}{l}\text { Angle }\left(^{\circ}\right) \\
\mathrm{H}-\mathrm{O}-\mathrm{H}\end{array}$} & \multicolumn{3}{|c|}{ Vibrational mode $\left(\mathrm{cm}^{-1}\right)$} \\
\hline & $\mathrm{O}-\mathrm{Fe}$ & $\mathrm{O}-\mathrm{H}(1)$ & $\mathrm{O}-\mathrm{H}(2)$ & & Asymmetric & Symmetric & Bending \\
\hline$G a s^{*}$ & - & 0.973 & 0.973 & 104.481 & 3824 & 3685 & 1588 \\
\hline $\mathrm{Ti}$ & 2.177 & 0.977 & 0.977 & 106.107 & 3749 & 3629 & 1565 \\
\hline $\mathrm{V}$ & 2.153 & 0.976 & 0.977 & 106.356 & 3753 & 3632 & 1566 \\
\hline $\mathrm{Cr}$ & 2.141 & 0.977 & 0.977 & 106.124 & 3750 & 3626 & 1570 \\
\hline $\mathrm{Mn}$ & 2.140 & 0.977 & 0.977 & 105.808 & 3745 & 3628 & 1565 \\
\hline $\mathrm{Fe}$ & 2.106 & 0.977 & 0.977 & 106.109 & 3752 & 3626 & 1570 \\
\hline $\mathrm{Co}$ & 2.124 & 0.976 & 0.977 & 106.464 & 3760 & 3640 & 1576 \\
\hline $\mathrm{Ni}$ & 2.115 & 0.976 & 0.977 & 106.522 & 3758 & 3638 & 1568 \\
\hline
\end{tabular}


Table S4. Bader charge analysis results of bare $\mathrm{X} @ \mathrm{Fe}_{12}$ (marked as *) and $\mathrm{X} @ \mathrm{Fe}_{12}-\mathrm{H}_{2} \mathrm{O}$ (marked as * $\mathrm{H}_{2} \mathrm{O}$ ). Charges are shown in |e|. The corresponding number of Fe atom and $\mathrm{H}$ atom can be seen in Figure S7(c). Bader charge analysis result of gas water are added under bare clusters.

\begin{tabular}{|c|c|c|c|c|c|c|c|c|c|c|c|c|c|c|}
\hline & \multicolumn{2}{|c|}{$\mathrm{Ti} @ \mathrm{Fe}_{12}$} & \multicolumn{2}{|c|}{$\mathrm{V} @ \mathrm{Fe}_{12}$} & \multicolumn{2}{|c|}{$\mathrm{Cr} @ \mathrm{Fe}_{12}$} & \multicolumn{2}{|c|}{$\mathrm{Mn} @ \mathrm{Fe}_{12}$} & \multicolumn{2}{|c|}{$\mathrm{Fe}_{13}$} & \multicolumn{2}{|c|}{$\mathrm{Co@Fe} \mathrm{Fe}_{12}$} & \multicolumn{2}{|c|}{$\mathrm{Ni@Fe}{ }_{12}$} \\
\hline & * & ${ }^{*} \mathrm{H}_{2} \mathrm{O}$ & * & ${ }^{*} \mathrm{H}_{2} \mathrm{O}$ & * & ${ }^{*} \mathrm{H}_{2} \mathrm{O}$ & * & ${ }^{*} \mathrm{H}_{2} \mathrm{O}$ & * & ${ }^{*} \mathrm{H}_{2} \mathrm{O}$ & * & ${ }^{*} \mathrm{H}_{2} \mathrm{O}$ & * & ${ }^{*} \mathrm{H}_{2} \mathrm{O}$ \\
\hline $\mathrm{Fe} 1$ & -0.06 & -0.13 & -0.15 & -0.10 & -0.04 & -0.06 & -0.02 & -0.03 & 0.00 & -0.04 & 0.01 & -0.03 & 0.02 & -0.02 \\
\hline $\mathrm{Fe} 2$ & -0.06 & -0.12 & -0.19 & -0.10 & 0.04 & -0.06 & -0.02 & -0.03 & -0.01 & -0.04 & 0.00 & -0.03 & 0.02 & 0.00 \\
\hline $\mathrm{Fe} 3$ & -0.10 & -0.08 & -0.17 & -0.10 & -0.03 & -0.05 & -0.02 & -0.04 & 0.00 & -0.03 & 0.02 & -0.01 & 0.01 & -0.02 \\
\hline $\mathrm{Fe} 4$ & -0.10 & -0.09 & -0.08 & -0.06 & -0.03 & -0.05 & -0.02 & -0.04 & 0.00 & -0.01 & 0.01 & 0.00 & 0.02 & 0.00 \\
\hline Fe5 & -0.06 & -0.08 & -0.08 & -0.06 & -0.02 & -0.04 & -0.03 & -0.05 & 0.00 & -0.10 & 0.00 & -0.01 & 0.01 & -0.01 \\
\hline Fe6 & -0.11 & -0.07 & -0.08 & -0.04 & -0.03 & -0.04 & -0.02 & -0.03 & 0.00 & 0.01 & 0.01 & 0.00 & 0.01 & 0.01 \\
\hline $\mathrm{Fe} 7$ & -0.06 & -0.06 & -0.07 & -0.04 & -0.03 & -0.05 & -0.03 & -0.04 & 0.00 & -0.02 & 0.01 & 0.00 & 0.02 & 0.01 \\
\hline Fe8 & -0.06 & -0.12 & -0.17 & -0.10 & -0.04 & -0.05 & -0.02 & -0.04 & 0.00 & -0.01 & 0.01 & 0.00 & 0.02 & 0.00 \\
\hline $\mathrm{Fe} 9$ & -0.05 & -0.12 & -0.19 & -0.11 & -0.04 & -0.05 & -0.02 & -0.03 & 0.01 & -0.03 & 0.02 & -0.01 & 0.02 & 0.00 \\
\hline Fe10 & -0.09 & -0.07 & -0.13 & -0.08 & -0.05 & -0.06 & -0.02 & -0.03 & 0.00 & -0.02 & 0.01 & 0.00 & 0.02 & 0.00 \\
\hline $\mathrm{Fe}_{\mathrm{para}}$ & -0.09 & -0.11 & -0.12 & -0.05 & -0.02 & -0.02 & -0.02 & -0.02 & 0.00 & 0.03 & 0.01 & 0.04 & 0.03 & 0.06 \\
\hline $\mathrm{Fe}_{\mathrm{ads}}$ & -0.10 & 0.07 & -0.11 & 0.13 & -0.03 & 0.14 & -0.02 & 0.13 & 0.00 & 0.17 & 0.00 & 0.20 & 0.02 & 0.24 \\
\hline$X$ & 0.94 & 0.96 & 1.54 & 0.67 & 0.32 & 0.39 & 0.26 & 0.22 & 0.00 & -0.02 & -0.11 & -0.31 & -0.22 & -0.25 \\
\hline 0 & -1.22 & -1.26 & -1.22 & -1.27 & -1.22 & -1.31 & -1.22 & -1.26 & -1.22 & -1.27 & -1.22 & -1.31 & -1.22 & -1.30 \\
\hline $\mathrm{H} 1$ & 0.63 & 0.65 & 0.63 & 0.65 & 0.63 & 0.67 & 0.63 & 0.64 & 0.63 & 0.69 & 0.63 & 0.67 & 0.63 & 0.66 \\
\hline $\mathrm{H} 2$ & 0.59 & 0.63 & 0.59 & 0.64 & 0.59 & 0.66 & 0.59 & 0.64 & 0.59 & 0.62 & 0.59 & 0.68 & 0.59 & 0.68 \\
\hline $\begin{array}{c}\text { Sum of } \\
\mathrm{H}_{2} \mathrm{O}\end{array}$ & 0 & 0.02 & 0 & 0.02 & 0 & 0.02 & 0 & 0.02 & 0 & 0.04 & 0 & 0.04 & 0 & -0.04 \\
\hline
\end{tabular}


Table S5. Geometrical parameters of the transition state for water dissociation on $\mathrm{X} @ \mathrm{Fe}_{12}$.

\begin{tabular}{ccccc}
\hline Doping-atom & $\mathrm{E}_{\mathrm{a}}(\mathrm{eV})$ & $\mathrm{E}_{\mathrm{r}}(\mathrm{eV})$ & $\mathrm{O}-\mathrm{H}$ & $\mathrm{H}-\mathrm{Fe}$ \\
\hline $\mathrm{Ti}$ & 0.42 & -0.75 & 1.417 & 1.696 \\
$\mathrm{~V}$ & 0.40 & -0.88 & 1.417 & 1.725 \\
$\mathrm{Cr}$ & 0.41 & -0.86 & 1.411 & 1.747 \\
$\mathrm{Mn}$ & 0.42 & -0.85 & 1.411 & 1.752 \\
$\mathrm{Fe}$ & 0.36 & -0.98 & 1.399 & 1.712 \\
$\mathrm{Co}$ & 0.36 & -1.09 & 1.388 & 1.731 \\
$\mathrm{Ni}$ & 0.30 & -1.16 & 1.388 & 1.745 \\
\hline
\end{tabular}

Table S6. Preferred magnetic states $\left(\mu_{\mathrm{B}}\right)$ of the studied center-doped X@ $\mathrm{Fe} \mathrm{e}_{12}$ systems.

\begin{tabular}{cccc}
\hline & bare & $* \mathrm{H}_{2} \mathrm{O}$ & $* \mathrm{OH}+{ }^{*} \mathrm{H}$ \\
\hline $\mathrm{Ti} @ \mathrm{Fe}_{12}$ & 38 & 38 & 38 \\
$\mathrm{~V} @ \mathrm{Fe}_{12}$ & 37 & 37 & 37 \\
$\mathrm{Cr} @ \mathrm{Fe}_{12}$ & 36 & 36 & 36 \\
$\mathrm{Mn} @ \mathrm{Fe}_{12}$ & 35 & 35 & 35 \\
$\mathrm{Fe}_{13}$ & 44 & 42 & 42 \\
$\mathrm{Co} @ \mathrm{Fe}_{12}$ & 43 & 43 & 41 \\
$\mathrm{Ni} @ \mathrm{Fe}_{12}$ & 42 & 42 & 40 \\
\hline
\end{tabular}


Table S7. Preferred magnetic states $\left(\mu_{\mathrm{B}}\right)$ of the studied center-doped $\mathrm{X} @ \mathrm{Fe}_{12}$ landing onto defected graphene (DG) systems.

\begin{tabular}{cccc}
\hline & bare & $* \mathrm{H} 2 \mathrm{O}$ & $* \mathrm{OH}+{ }^{*} \mathrm{H}$ \\
\hline $\mathrm{Ti} @ \mathrm{Fe}_{12} / \mathrm{DG}$ & 35 & 35 & 33 \\
$\mathrm{Fe}_{13} \mathrm{DG}$ & 34 & 34 & 34 \\
$\mathrm{Ni} @ \mathrm{Fe}_{12} / \mathrm{DG}$ & 34 & 34 & 34 \\
\hline
\end{tabular}

As the shell-doped $\mathrm{Mn}-\mathrm{Fe}_{12}$ and $\mathrm{Ni}-\mathrm{Fe}_{12}$ clusters are more stable than the corresponding centerdoped clusters, we also considered the water adsorption and dissociation on the shell-doped clusters. There are four possible adsorption sites on $\mathrm{X}-\mathrm{Fe}_{12}$, shown as doping atom $\mathrm{X}$, ortho-Fe, meta-Fe and para-Fe. We analyzed the interaction between water and $\mathrm{X}-\mathrm{Fe}_{12}(\mathrm{X}=\mathrm{Ti}$ and $\mathrm{Ni})$, finding the charge transfer occurs from $\mathrm{Ti}$ to $\mathrm{Fe}$, and conversely from Fe to $\mathrm{Ni}$ (supported by Bader charge analysis). The adsorption energies, reaction barriers and reaction energies are shown in TableS6. The adsorption energies for water on different sites on $\mathrm{Ni}-\mathrm{Fe}_{12}$ were almost the same, although the activation energy at $\mathrm{Ni}$ atom is the largest. For Ti-Fe ${ }_{12}$, the adsorption energy of water adsorbed on Ti site is largest, however, the activation energy is the smallest which is only $0.10 \mathrm{eV}$. The activation energy at orthoand meta-Fe atoms of both $\mathrm{Ni}-\mathrm{Fe}_{12}$ and $\mathrm{Ti}-\mathrm{Fe}_{12}$ are smaller than para-Fe atom which is almost the same as the $\mathrm{E}_{\mathrm{a}}$ on $\mathrm{Fe}_{13}$. The PDOS analyses results of $\mathrm{H}_{2} \mathrm{O}$ adsorb on the ortho-Fe, meta-Fe and para-Fe of $\mathrm{X}-\mathrm{Fe}_{12}$ are shown in Figure S9. 
Table S8. Properties of shell-doped X-Fe 12 clusters.

\begin{tabular}{cccccc}
\hline & & $\mathrm{O}-\mathrm{M}(\AA)$ & $\mathrm{E}_{\mathrm{ads}}(\mathrm{eV})$ & $\mathrm{E}_{\mathrm{a}}(\mathrm{eV})$ & $\mathrm{E}_{\mathrm{r}}(\mathrm{eV})$ \\
\hline \multirow{2}{*}{$\mathrm{Ni}^{-\mathrm{Fe}_{12}}$} & $\mathrm{Ni}$ & 2.034 & -0.65 & 0.50 & -1.00 \\
& ortho-Fe & 2.113 & -0.62 & 0.30 & -0.99 \\
& meta-Fe & 2.104 & -0.64 & 0.29 & -1.04 \\
& para-Fe & 2.103 & -0.64 & 0.35 & -0.96 \\
& $\mathrm{Ti}$ & 2.178 & -0.91 & 0.10 & -1.51 \\
& ortho-Fe & 2.141 & -0.51 & 0.29 & -1.64 \\
& meta-Fe & 2.129 & -0.50 & 0.30 & -1.03 \\
& para-Fe & 2.174 & -0.38 & 0.36 & -1.20 \\
\hline
\end{tabular}
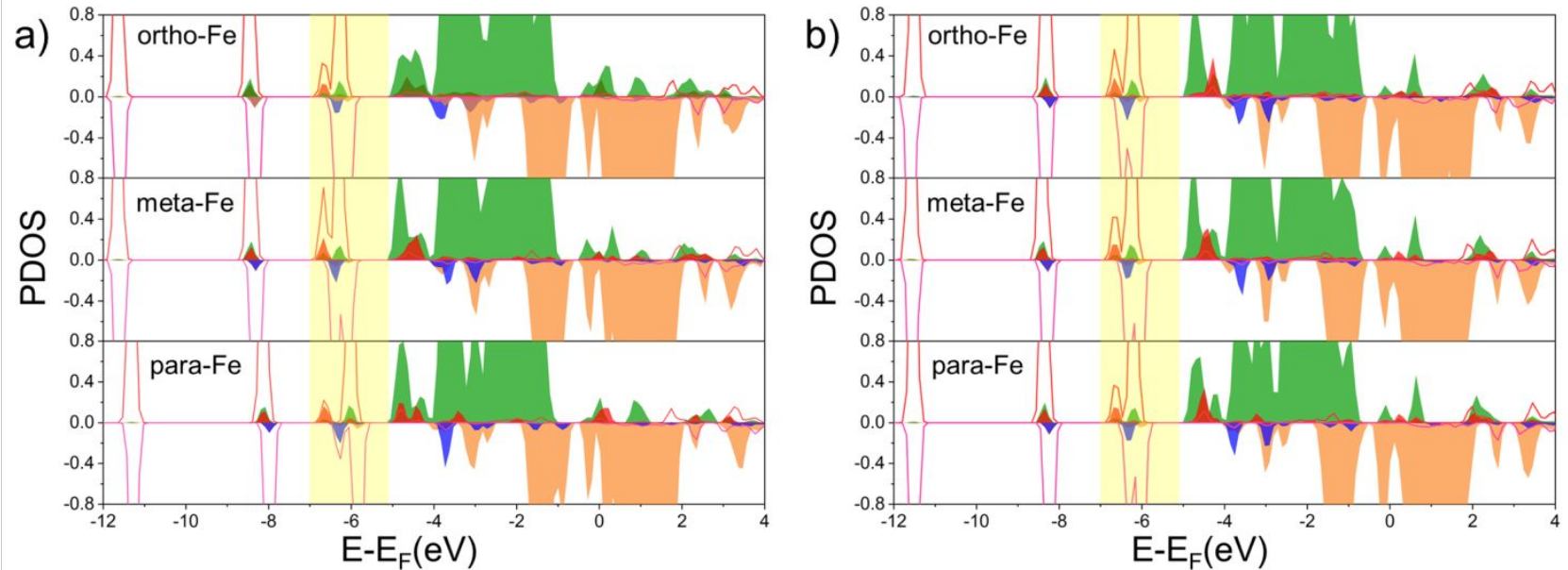

Figure S9. The PDOS for the $\mathrm{H}_{2} \mathrm{O}$ and the adsorbed $\mathrm{Fe}$ atom of different site in a) Ti-Fe $\mathrm{F}_{12}-\mathrm{H}_{2} \mathrm{O}$ and b) $\mathrm{Ni}-\mathrm{Fe}_{12}-\mathrm{H}_{2} \mathrm{O}$ systems. The area filled with green and orange represent the spin up (alpha) and spin down (beta) states of d orbitals of Fe atoms, respectively. The area filled with red and blue represent the spin up (alpha) and spin down (beta) states of s orbitals of Fe atoms, respectively. Red and purple solid line represent the $\mathrm{p}$ orbital of $\mathrm{O}$ atom. The Fermi level is set to be zero. 